\title{
Spin Seebeck Effect in Neodymium Iron Garnet Multilayers
}

\author{
Shashank Tyagi, ${ }^{1}$ Taito Maeda, ${ }^{2}$ Keisuke Kimura, ${ }^{1}$ Surbhi Gupta, ${ }^{1}$ \\ Kengo Kishimoto, ${ }^{2}$ Tsuyoshi Koyanagi, ${ }^{2}$ Hironori Asada, ${ }^{2}$ and Yasuhiro Fukuma ${ }^{1 *}$ \\ ${ }^{1}$ Department of Computer Science and Electronics, Kyushu Institute of Technology, \\ 680-4 Kawazu, Iizuka 820-8502, Japan \\ ${ }^{2}$ Department of Electronic Devices and Engineering, Graduate School of Science and Engineering, \\ Yamaguchi University, Ube 755-8611, Japan
}

(Received February 18, 2019; accepted June 3, 2019)

Keywords: spin caloritronics, spin Seebeck effect, spin current, thermoelectric films, nanoscale sensing

Nanoscale sensing applications require significant power to operate, which can be largely supplied through the efficient management of omnipresent heat. The spin Seebeck effect (SSE) is rather a fresh energy harvesting phenomenon that enables the conversion of a temperature gradient across magnetic materials into spin current. This spin current can further be converted into charge current by adjoining heavy metals to the magnet. In this study, we fabricated the thermoelectric multilayer films of bismuth-substituted neodymium iron garnet $\left(\mathrm{Nd}_{2} \mathrm{Bi}_{1} \mathrm{Fe}_{5} \mathrm{O}_{12}\right)(\mathrm{NIG})$ as well as gallium and bismuth co-substituted neodymium iron garnet $\left(\mathrm{Nd}_{2} \mathrm{Bi}_{1} \mathrm{Fe}_{4} \mathrm{Ga}_{1} \mathrm{O}_{12}\right)$ (NIGG) on (111)-oriented gallium gadolinium garnet substrates by metal organic decomposition. The thicknesses of the NIG and NIGG multilayers are varied to control the magnetic properties. The observed spin Seebeck signal is found to be directly dependent on the garnet/heavy metal interface and tends to decrease in intensity with increasing amount of NIGG in the garnet multilayers. Our results emphasize the importance of magnet/ heavy metal interface properties for designing SSE-based sensors.

\section{Introduction}

Energy harvesting and its efficient utilization are crucial pillars of a sustainable future, for which thermoelectric, piezoelectric, and photoelectric effect-based technologies have been explored to scavenge omnipresent heat, vibration, and electromagnetic waves from the environment. These are intended to be implemented in wireless embedded sensors for IoT applications, such as recharging microbatteries, monitoring pressure in automobiles, efficient functioning of large-scale data centers, and running security systems in household/office conditions. Thus, there is a continuous demand for smart transducers that can perform effective thermal management with real-time controllability and higher sensitivity. Among various possibilities, spin caloritronics has attracted considerable attention in recent years owing to micro-macroscale components and integration that show great potential in the development of low-power electronic devices. ${ }^{(1)}$ Devices based on the spin Seebeck effect (SSE) have

*Corresponding author: e-mail: fukuma@cse.kyutech.ac.jp

https://doi.org/10.18494/SAM.2019.2335 
various applications, such as temperature sensing, ${ }^{(2)}$ two-dimensional position sensing, ${ }^{(3)}$ and thermoelectric power generation. The advantage of extending the SSE to heat-flow sensor applications lies in the uncomplicated structure of SSE-based sensors ${ }^{(4)}$ and exhibitance in a variety of energy-harvesting ferromagnetic materials ranging from metals, ${ }^{(5)}$ semiconductors, ${ }^{(6)}$ to insulators. ${ }^{(7,8)}$ For example, Bi-substituted iron insulator garnets are of interest owing to their strong spin-orbit interaction to generate pure spin currents with the flexible control of magnetic properties by changing the composition..$^{(9,10)}$ As mentioned above, the simple experimental geometry of SSE-based sensors further allows two independent paths for heat and electric currents; thus, two different materials can be optimized separately for the implementation of thin films even over large surfaces also. In the present work, a technique that combines metal organic decomposition (MOD) and spin coating, is employed to deposit $\mathrm{Nd}_{2} \mathrm{BiFe}_{5} \mathrm{O}_{12}$ (NIG)- and $\mathrm{Nd}_{2} \mathrm{BiFe}_{4} \mathrm{Ga}_{1} \mathrm{O}_{12}$ (NIGG)-based multilayer structures as it is not only a low-cost but also an easy scaling process by simply increasing the area of coating. ${ }^{(4)}$ In our previous work, the SSE in $\mathrm{Nd}_{2} \mathrm{BiFe}_{5-x} \mathrm{Ga}_{x} \mathrm{O}_{12}$ with various $\mathrm{Ga}$ compositions was studied and the SSE signal intensity was found to increase with saturation magnetization. ${ }^{(11)}$ However, it was not clear if the change in the degree of magnetization enhances magnon generation as well as transport in garnets or increases the mixing conductance that determines the spin current injected into the adjacent Pt layer, which converts spin current to charge current by means of the inverse spin Hall effect. Here, the detailed mechanism of the enhanced SSE was still not clarified. Therefore, in this study, to understand the effects of magnetization and mixing conductance on the SSE, multilayer samples of $\mathrm{Nd}_{2} \mathrm{BiFe}_{5} \mathrm{O}_{12}$ (NIG) and $\mathrm{Nd}_{2} \mathrm{BiFe}_{4} \mathrm{Ga}_{1} \mathrm{O}_{12}$ (NIGG) are designed by varying the thickness ratio and stack ordering in order to identify the crucial factors that can improve further SSE signal and in turn the sensitivity of garnet-based thin-film sensors.

\section{Sample Fabrication Process}

The garnet multilayers used in this study are fabricated in a clean room facility of the Nanofabrication Platform at Yamaguchi University. To fabricate a neodymium iron garnet layer, $10 \mu 1$ of MOD solution made from carboxylates of a desired composition is dropped on a (111)-oriented gallium gadolinium garnet (GGG) substrate using a micropipette and then spin-coated at $3000 \mathrm{rpm}$ for $30 \mathrm{~s}$. The layer is then dried and preannealed at 100 and $450{ }^{\circ} \mathrm{C}$, respectively, for $10 \mathrm{~min}$ each. The thickness of one layer is found to be approximately $40 \mathrm{~nm}$. This cycle is repeated five times to obtain the desired thickness of $200 \mathrm{~nm}$. The number of cycles for the respective garnet layer (NIG or NIGG) is adjusted on the basis of their studied thickness, that is, for a NIGG $(80 \mathrm{~nm}) / \mathrm{NIG}(120 \mathrm{~nm})$ sample, NIGG solution is coated twice, while NIG solution is applied three times. Finally, all samples are annealed at $600{ }^{\circ} \mathrm{C}$ in a closed oven for $3 \mathrm{~h}$ to achieve the desired crystalline phase of multilayer samples as shown in Fig. 1. In a similar manner, reverse stacks of multilayer structures are also fabricated.

Then, a 10-nm-thick Pt layer is deposited using a DC sputtering system [Fig. 2(a)] at a base pressure of $2.28 \times 10^{-7} \mathrm{~Pa}$ and an Ar deposition pressure of $1 \mathrm{~Pa}$ at $50 \mathrm{~W}$ on all multilayer samples. Thereafter, a strip $\left(2 \times 7 \mathrm{~mm}^{2}\right)$ of the top Pt layer is designed by photolithography to 


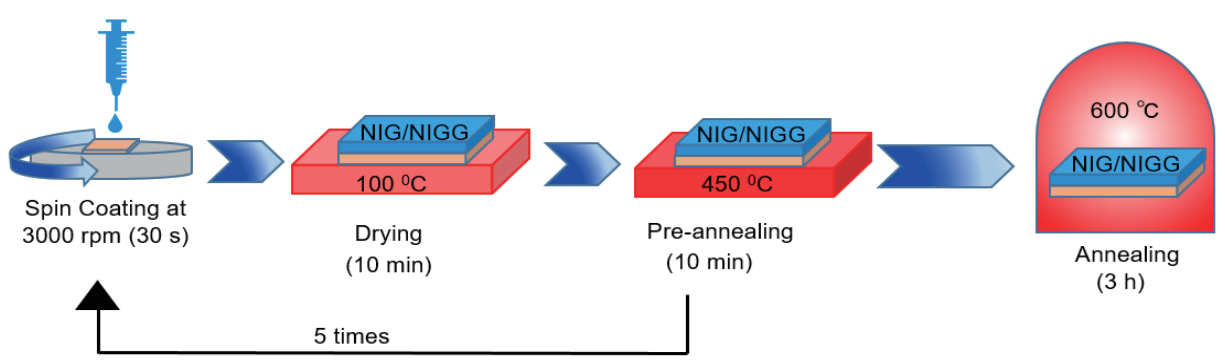

Fig. 1. (Color online) Fabrication process for NIG/NIGG multilayers using spin coating.

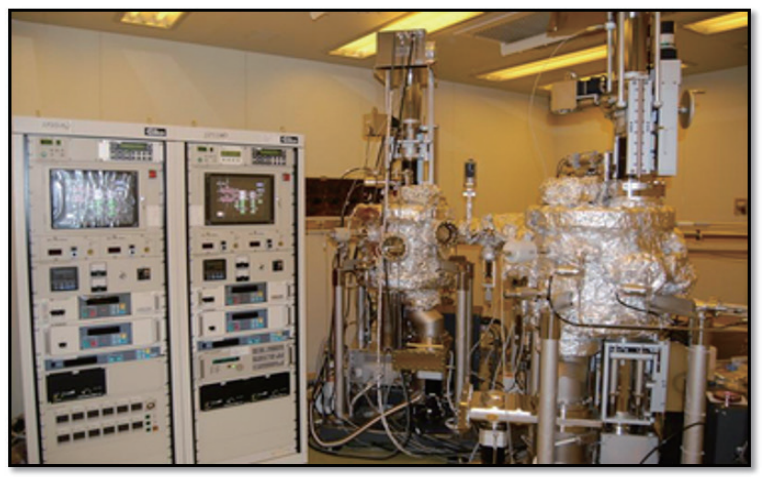

(a)

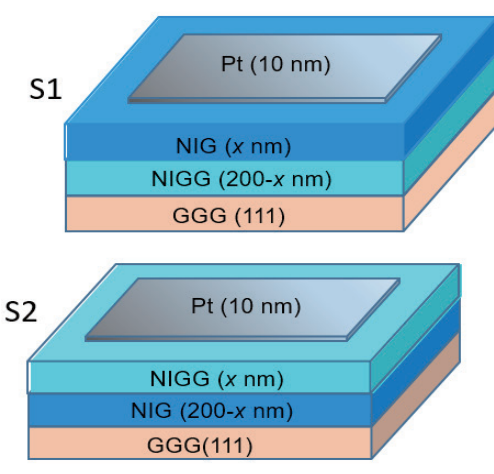

(b)

Fig. 2. (Color online) (a) Sputtering system in Yamaguchi University. (b) Schematic of neodymium iron garnet multilayer series $\mathrm{S} 1$ and $\mathrm{S} 2$.

obtain the desired structure to measure the SSE signal. Figure 2(b) shows the series structure used in this study. The total thickness of the NIG/NIGG multilayer is fixed to be $200 \mathrm{~nm}$, but the thickness ratio of NIG to NIGG is varied to control the average magnetization $\left(M_{\text {ave }}\right)$ in the garnet multilayers. Hereafter, the series of GGG/NIGG(200-x) nm/NIG(x) nm/Pt and GGG/ $\mathrm{NIG}(200-x) \mathrm{nm} / \mathrm{NIGG}(x) \mathrm{nm} / \mathrm{Pt}$ are referred to as S1 and S2, respectively. Series S2 with a reverse stacking order of NIG and NIGG to the adjacent Pt layer is designed to determine the effect of the interface or in other words the spin-mixing conductance of Pt/NIG and Pt/NIGG interfaces on SSE signal.

\section{Results and Discussion}

The crystallographic property of coating layers is examined using an X-ray diffractometer with a $\mathrm{Cu} \mathrm{K} \mathrm{K}_{\alpha}$ source $(1.54 \AA$ ). Clear X-ray diffraction (XRD) patterns with a (444) peak from NIG and NIGG are observed for all the samples, where Fig. 3(a) shows no significant change in characteristic peak position for 200-nm-thick NIG (S1) and NIGG (S2) multilayer samples, implying a similar crystal structure with a lattice constant of approximately $12.58 \AA$. Therefore, 


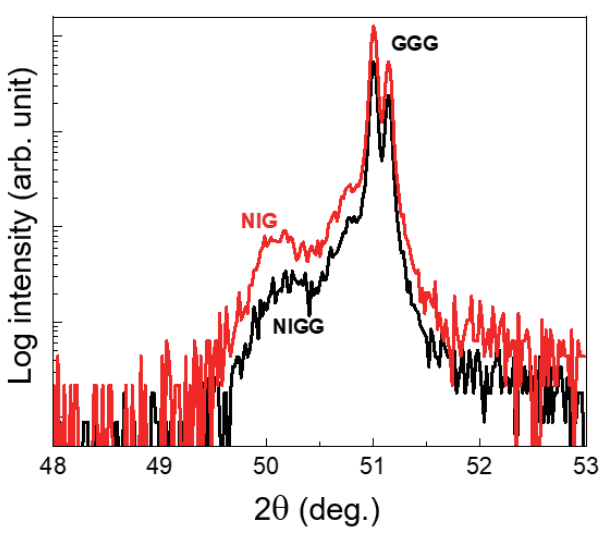

(a)

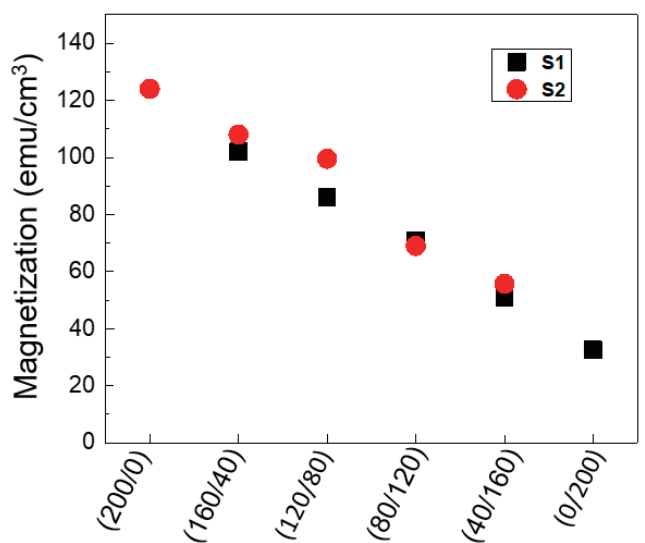

(b)

Fig. 3. (Color online) (a) XRD patterns of 200-nm-thick NIG (S1) and NIGG (S2) layers. (b) Mave values of different multilayers. Numbers in parentheses on the abscissa represent the respective thicknesses of NIG/NIGG layers in $\mathrm{nm}$.

it is considered that NIG/NIGG multilayers with different thickness ratios and their reverse layer structures do not affect crystallographic properties. Furthermore, magnetization curves are traced by applying an in-plane magnetic field swept from +2500 to -2500 Oe. All the samples exhibited a clear hysteresis loop, suggesting that the magnetic easy axis lies in the plane. The saturation value of average magnetization $\left(M_{a v e}\right)$ as a function of the layer composition is shown in Fig. 3(b), where its linear decrease with increasing NIGG layer thickness can be observed. This decrease is attributed to replacement of magnetic iron ions by gallium ions in NIGG layer.

Next, for measurements of SSE-voltage signals, the temperature gradient is monitored and maintained continuously across the sample using a temperature controller and an external magnetic field is applied as shown in Fig. 4(a). The electrical signal generated by the temperature gradient is then detected as a voltage between the electrodes along the top Pt layer strip using a digital multimeter. A clear hysteresis loop in the magnetic field dependence of the voltage is depicted in Fig. 4(b), where the voltage sign reverses on reversing the direction of the applied external magnetic field because the spin polarization vector (along the external magnetic field), the spin current direction (i.e., from garnet to Pt), and the charge current direction are always orthogonal to each other. ${ }^{(12)}$

To neglect the effect of different electrode contact resistances in all samples, the generated spin Seebeck current $\left(V_{S S E} / R_{P t}\right)$ is rather compared among the samples. Figure 5(a) shows the spin Seebeck current $\left(V_{S S E} / R_{P t}\right)$ values for all the samples. The current tends to decrease with increasing amount of NIGG in both garnet series S1 and S2.

To further confirm that voltage signals purely originate from the SSE, temperature gradient $(\Delta T)$-dependent SSE measurements are also performed for all the samples. Figure 5(b) displays voltage curves whose intensity is found to be linearly dependent on $\Delta T$ (inset) for the S1: NIGG(40 nm)/NIG(160 nm) sample. We observed a similar behavior for other samples as well.

The observed behavior of $V_{S S E}$ may be attributed to the decrease in $M_{\text {ave }}$ with NIGG thickness as it further leads to a decrease in the thermal stability of the garnet bilayers; 


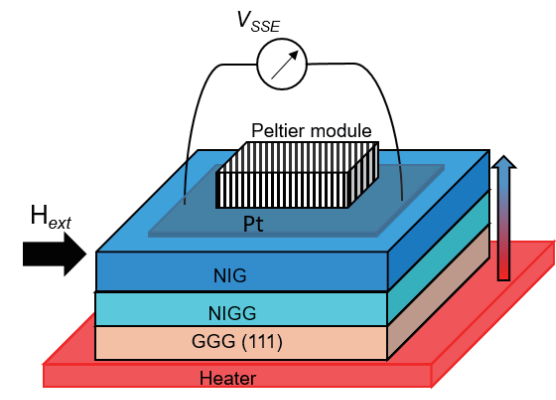

(a)

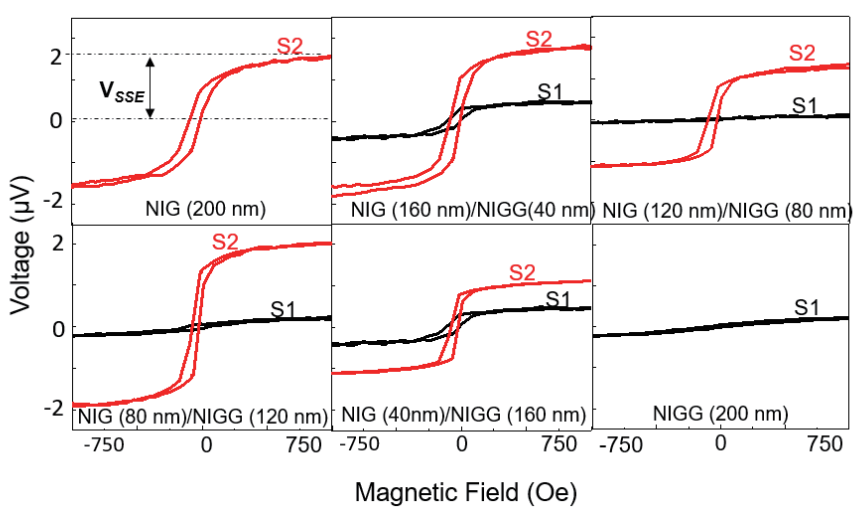

(b)

Fig. 4. (Color online) (a) Experimental setup for measuring SSE-voltage signals. (b) Voltage signal as a function of magnetic field for different multilayers.

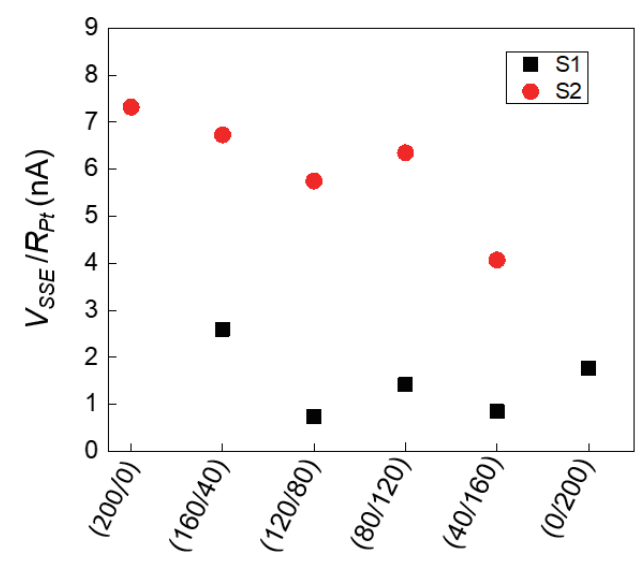

(a)

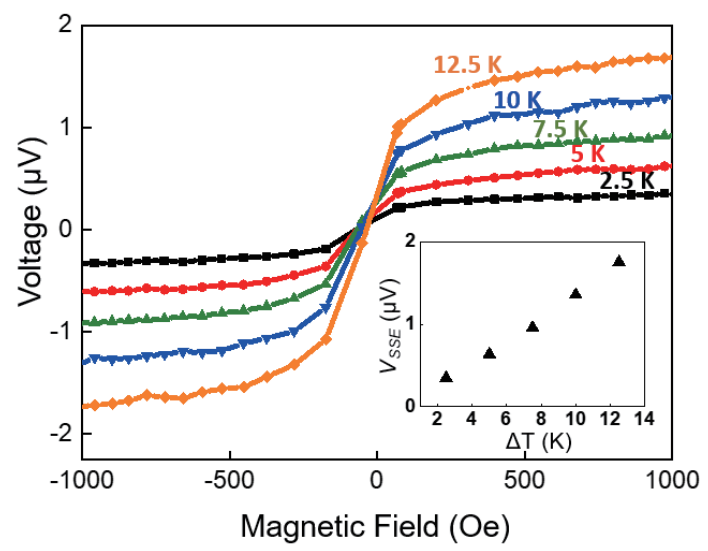

(b)

Fig. 5. (Color online) (a) Spin Seebeck currents of different multilayers. Numbers in parentheses on the abscissa represent the respective thicknesses of NIG/NIGG layers in $\mathrm{nm}$. (b) Voltage-field dependence for NIGG(40 nm)/ NIG(160 nm) multilayer at different $\Delta T$. The inset shows the linear variation in VSSE intensity with $\Delta \mathrm{T}$.

therefore, a large magnetization precession is expected for the given thermal gradient, which is expected to increase the spin current generation. ${ }^{(13)}$ To better understand the spin current and magnon transport characteristics in relation to $M_{\text {ave }}$, further studies are required. On the other hand, the amount of spin Seebeck current is found to be clearly changed by the top layer of neodymium iron garnet in present studies of structures. As series S1 with top layer of NIG showed a spin Seebeck current, which is fourfold higher than that of NIGG. The mixing conductance of NIG/Pt is also estimated to be higher than that of NIGG/Pt because of the higher moment density at the interface, causing a significant increase in the intensity of the spin Seebeck signal. 


\section{Conclusions}

We fabricated thermoelectric samples consisting of NIGG and NIG multilayers on GGG substrates by MOD, and found that the generated average spin Seebeck signal is fourfold higher in intensity in the case of multilayers with a NIG/Pt interface than in the case of a NIGG/Pt interface. Also, the spin Seebeck signal intensity tends to decrease as the thickness of the NIGG layer in the garnet increases, irrespective of stack ordering. We believe that this experimental study will further clarify the factors crucial to improving SSE signals in granet-based sensors to achieve higher controllability and sensitivity.

\section{Acknowledgments}

This work was supported by JSPS KAKENHI Grant Number $18 \mathrm{H} 01862$ and Nippon Sheet Glass Foundation for Materials Science and Engineering.

\section{References}

1 G. E. W. Bauer, E. Saitoh, and B. J. van Wees: Nat. Mater. 11 (2012) 391. https://doi.org/10.1038/nmat3301

2 Tianjun Liao, Zhoulin Ye, and Jincan Chen: IEEE Trans. Electron Devices 64 (2017) 2655. https://doi. org/10.1109/ted.2017.2691762

3 K. Uchida, A. Kirihara, M. Ishida, R. Takahashi, and E. Saitoh: Jpn. J. Appl. Phys. 50 (2011) 120211. https:// doi.org/10.1143/JJAP.50.120211

4 A. Kirihara, K. Uchida, Y. Kajiwara, M. Ishida, Y. Nakamura, T. Manako, E. Saitoh, and S. Yorozu: Nat. Mater. 11 (2012) 686. https://doi.org/10.1038/nmat3360

5 K. Uchida, T. Ota, K. Harii, K. Ando, H. Nakayama, and E. Saitoh: J. Appl. Phys. 107 (2010) 09A951. https:// doi.org/10.1063/1.3357413

6 C. M. Jaworski, J. Yang, S. Mack, D. D. Awschalom, J. P. Heremans, and R. C. Myers: Nat. Mater. 9 (2010) 898. https://doi.org/10.1038/nmat2860

7 K. Uchida, J. Xiao, H. Adachi, J. Ohe, S. Takahashi, J. Ieda, T. Ota, Y. Kajiwara, H. Umezawa, H. Kawai, G. E. W. Bauer, S. Maekawa, and E. Saitoh: Nat. Mater. 9 (2010) 894. https://doi.org/10.1038/nmat2856

8 K. Uchida, H. Adachi, T. Ota, H. Nakayama, S. Maekawa, and E. Saitoh: Appl. Phys. Lett. 97 (2010) 172505. https://doi.org/10.1063/1.3507386

9 M. Sasaki, G. Lou, Q. Liu, M. Ninoniya, T. Kato, S. Iwata, and T. Ishibashi: Jpn. J. Appl. Phys. 55 (2016) 055501. https://doi.org/10.7567/JJAP.55.055501

10 J. Yamakita, G. Lou, M. Ishikawa, T. Kato, S. Iwata, and T. Ishibashi: Jpn. J. Appl. Phys. 57 (2018) 09TC01. https://doi.org/10.7567/JJAP.57.09TC01

11 H. Asada, A. Kuwahara, K. Sueyasu, T. Ishibashi, Q. Liu, G. Lou, K. Kishimoto, and T. Koyanagi. J. Appl. Phys. 117 (2015) 17C724. https://doi.org/10.1063/1.4914361

12 J. Xiao, G. E. W. Bauer, K. Uchida, E. Saitoh, and S. Maekawa: Phys. Rev. B 81 (2010) 214418. https://doi. org/10.1103/PhysRevB.81.214418

13 S. Gupta, R. Medwal, D. Kodama, K. Kondou, Y. Otani, and Y. Fukuma: Appl. Phys. Lett. 110 (2017) 022404. https://doi.org/10.1063/1.4973704 